\title{
Tendances observées dans la surveillance de la coqueluche en Colombie-Britannique (Canada) sur une période de 20 ans, de 1993 à 2013
}

\author{
Chambers $\mathrm{C}^{1}$, Skowronski $\mathrm{DM}^{1}{ }^{1}$, Hoang $\mathrm{L}^{2}$, Guiyun $\mathrm{Li} \mathrm{H}^{1}$, Fritz $\mathrm{CE}^{1}$ Gustafson $\mathbf{R}^{3}$, \\ Murti $\mathbf{M}^{4}$, Reid $\mathrm{A}^{5}$, Parker $\mathrm{R}^{6}$ et Bowering $\mathrm{D}^{7}$
}

1 British Columbia Centre for Disease Control, Provincial Health Services Authority, Vancouver (C.-B.)

2 Laboratoire de santé publique et de référence en microbiologie de la Colombie-Britannique, Provincial Health Services Authority, Vancouver (C.-B.)

3 Vancouver Coastal Health Authority, Vancouver (C.-B.)

$4 \quad$ Fraser Health Authority, Surrey (C.-B.)

$5 \quad$ Vancouver Island Health Authority, Victoria (C.-B.)

$6 \quad$ Interior Health Authority, Kelowna (C.-B.)

$7 \quad$ Northern Health Authority, Prince George (C.-B.)

* $\quad$ Auteure-ressource : danuta.skowronski@bccdc.ca

\section{Résumé}

Objectif : Fournir une mise à jour sur la surveillance des tendances relatives à la coqueluche dans l'ensemble et par groupes d'âge en Colombie-Britannique (C.-B.), au Canada, sur une période de 20 ans, soit de 1993 à 2013.

Méthodes: Des données sur la surveillance des cas confirmés de coqueluche à l'échelle provinciale ont été extraites pour la période entre le 1er janvier 1993 et le 31 octobre 2013. Des taux d'incidence annuels et propres à l'âge ont été calculés à partir d'estimations dans les populations provinciales et régionales.

Résultats : La C.-B. a connu une épidémie importante de coqueluche à la fin des années 1990 et au début des années 2000, avec des taux d'incidence globaux variant entre 20 et 40 cas pour 100000 habitants et atteignant un sommet de plus de 200 cas pour 100000 préadolescents de 10 à 13 ans au cours de l'épidémie de l'année 2000. Entre 2005 et 2011, le taux d'incidence global a chuté à des niveaux historiquement bas variant entre 1 à 6 cas pour 100000 habitants. Cette faible activité a été suivie, en 2012, par une résurgence attribuable principalement aux éclosions dans les régions des basses-terres continentales de la C.-B., pendant laquelle l'incidence globale à l'échelle provinciale a atteint 10 cas pour 100000 habitants. L'incidence propre à l'âge la plus élevée en 2012 a été observée chez les nourrissons de moins de 1 an (64 cas pour 100000 habitants) et chez les enfants de 12 et 13 ans (entre 56 et 57 cas pour 100000 habitants), alors qu'un changement dans la répartition selon l'âge, soit une diminution du nombre des enfants d'âge préscolaire et une augmentation du nombre de préadolescents et d'adolescents, était observé depuis l'année 2000. L'incidence chez les adultes est restée à moins de 10 cas pour 100000 habitants pendant toute la période et était de 5 cas pour 100000 habitants en 2012. Le taux d'incidence provincial cumulatif global en 2013 était de 6 cas pour 100000 habitants, alors qu'une activité asynchrone continue était observée principalement sur l'île de Vancouver.

Conclusion : L'activité de la coqueluche en C.-B. a connu des fluctuations cycliques attendues. Un sommet d'incidence a été observé en 2012, principalement chez les nourrissons et chez les préadolescents et adolescents, mais à des niveaux inférieurs aux sommets précédents. Après une épidémie importante dans les années 1990 et au début des années 2000 et l'intégration d'un vaccin anticoquelucheux acellulaire au programme de vaccination systématique, l'immuno-épidémiologie de la coqueluche pourrait être encore en transition.

D'autres efforts de surveillance et d'évaluation sont requis pour déterminer les changements éventuels à apporter aux programmes de la Colombie-Britannique. 


\section{Introduction}

La coqueluche («toux coquelucheuse ») demeure une maladie endémique au Canada, comme partout ailleurs dans le monde, caractérisée par des points culminants survenant tous les 2 à 5 ans ${ }^{1}$. Les nourrissons de moins de 1 an présentent le risque le plus élevé de maladies graves, y compris d'hospitalisation, d'admission en unité de soins intensifs (USI) et de décès, le risque le plus élevé étant observé chez les très jeunes nourrissons de moins de 3 mois. ${ }^{2,3}$

Après l'introduction au Canada, en 1943, d'un programme d'immunisation systématique des enfants au moyen d'un vaccin anticoquelucheux à germes entiers, l'incidence de la coqueluche a diminué de plus de $90 \%$, passant d'une moyenne de 165 cas pour 100000 habitants entre 1935 et 1939 à 10 cas ou moins pour 100000 habitants vers la fin des années des 1980, puis à un niveau historiquement bas de 4 cas pour 100000 habitants en $1988 .{ }^{4-6}$ Toutefois, malgré la mise en place de programmes d'immunisation universels des enfants, l'incidence de la coqueluche a augmenté de façon spectaculaire au Canada au cours des années 1990 et au début des années 2000 et une tendance à l'augmentation des taux d'infection a été observée chez les enfants plus âgés ${ }^{4}$. Dans les régions les plus à l'ouest de la Colombie Britannique (B.-C.), l'incidence propre à l'âge au cours des sommets cycliques de 2000 et 2003 était pour la première fois considérablement plus élevée chez les préadolescents et les jeunes adolescents, avec des sommets d'incidence de 150 à 300 cas pour 100000 habitants dans ces groupes d'âge, et ont même dépassé les taux signalés chez les nourrissons ${ }^{7}$. Cette résurgence, ainsi que le changement observé simultanément dans le profil d'âge, ont été largement attribués à l'avancement de l'effet de cohorte découlant de l'efficacité sous-optimale (entre 20 et $60 \%$ ) du vaccin absorbé à germes entiers utilisé au Canada entre 1980 et $1997^{7-12}$, mais également à l'affaiblissement de l'immunité, à la sensibilisation accrue chez les cliniciens et aux améliorations apportées aux tests diagnostiques, soit l'inclusion d'un test basé sur la réaction en chaîne de la polymérase (RCP), considéré comme plus sensible. ${ }^{4,5,7-9,12}$

En réponse à l'évolution des tendances observées sur le plan de la coqueluche, la plupart des provinces et territoires canadiens, y compris la C.-B., sont passés dès 1997 à un vaccin acellulaire à 5 composants moins réactogène et plus efficace (efficacité de plus de $85 \%$ après 3 doses). ${ }^{10 ; 13}$ Depuis, un vaccin anticoquelucheux acellulaire est utilisé dans le cadre du programme d'immunisation systématique de la C.-B. chez les enfants âgés de 2, 4, 6 et 18 mois et pour la dose de rappel administrée aux enfants de la maternelle entre l'âge de 4 et 6 ans. Une autre dose de rappel pour les adolescents de la 9 e année (14 et 15 ans) a été introduite en C.-B. en 2004.,14

Après les importantes éclosions de coqueluche observées à la fin des années 1990 et au début des années 2000, pendant lesquelles l'incidence provinciale a atteint un sommet avec 20 à 40 cas pour 100000 habitants dans l'ensemble de la C.-B., l'activité de la coqueluche a chuté à de faibles niveaux variant entre 1 et 6 cas pour 100000 habitants entre 2005 et 2011. Toutefois, cette activité historiquement faible en C.-B. est survenue simultanément aux nombreux signalements d'une résurgence de l'activité de la coqueluche aux États-Unis ${ }^{3,15}$ principalement dans l'État de la Californie, également situé sur la côte ouest, où l'incidence globale d'environ 25 cas pour 100000 habitants observée en 2010 a été la plus élevée en plus de 50 ans. ${ }^{16}$

En 2012 et 2013, la C.-B. a connu d'autres sommets cycliques d'activité de la coqueluche dans certaines régions de la province. La présente mise à jour sur la surveillance décrit les tendances globales et par groupes d'âge pour la coqueluche en C.-B. sur une période de 20 ans, soit de 1993 à 2013. Cette perspective décrit les répercussions immuno-épidémiologiques à l'échelle des populations des épidémies précédentes ainsi que les variations des programmes d'immunisation qui pourraient avoir contribué aux tendances récemment observées pour la coqueluche. 


\section{Méthodes}

\section{Ensemble des données de surveillance}

La coqueluche est une maladie à déclaration obligatoire en C.-B. Les cas de coqueluche sont signalés au BC Centre for Disease Control par l'intermédiaire du Système d'information sur la santé publique intégré (SISP i, toutes les autorités sanitaires, à l'exception de la Vancouver Coastal Health Authority [VCHA]) ou du Primary Access Regional Information System (PARIS; VCHA seulement). Conformément aux exigences nationales en matière de signalement, le présent rapport décrit les tendances épidémiologiques fondées sur le signalement des cas confirmés seulement. Les cas confirmés comprennent les cas confirmés en laboratoire (culture ou réaction en chaîne de la polymérase) d'infections par Bordetella pertussis ou les liens épidémiologiques vers des cas confirmés en laboratoire accompagnés de symptômes correspondant à une infection par B. pertussis. ${ }^{17}$

La réaction en chaîne de la polymérase $(\mathrm{RCP})$ a été introduite en avril 1998 comme test diagnostique de routine, en plus de la culture de la coqueluche, par le Laboratoire de santé publique et de référence en microbiologie (PHMRL) de la C.-B., qui effectue la majorité des tests de dépistage de la coqueluche en C.-B. et signale plus de $85 \%$ de tous les cas confirmés détectés (sur le plan historique et au cours des récentes éclosions). ${ }^{7}$ Depuis mai 2000, la RCP est acceptée à l'échelle nationale, tout comme la culture, comme confirmation en laboratoire. Tous les cas de coqueluche confirmés en laboratoire (sans égard au laboratoire qui effectue le test) doivent être signalés à l'autorité provinciale de santé publique et sont ensuite signalés par l'intermédiaire du système SISPi/PARIS par les autorités sanitaires.

Les données sur la surveillance à l'échelle provinciale (âge, sexe et localisateurs géographiques) ont été extraites du système SISP-i/PARIS entre le 1er janvier 1993 et le 31 octobre 2013 pour tous les cas confirmés de coqueluche (diagnostic en laboratoire ou lien épidémiologique) signalés en C.-B. Des renseignements sur la surveillance accrue de l'éclosion de 2012 ont été résumés localement au sein des autorités sanitaires concernées (Fraser Health Authority [FHA] et VCHA), dont des renseignements sur les antécédents vaccinaux et les cas graves (p. ex. hospitalisation et décès). Chaque cas a été assigné à une autorité régionale de la santé, selon le service de santé local qui a effectué le signalement. L'analyse du nombre de demandes de tests soumises mensuellement au PHMRL et les taux de tests positifs pour la coqueluche étaient fondés sur des données extraites du système d'information sur les laboratoires Sunquest entre le 1er janvier 2011 et le 30 septembre 2013.

Comme les données ont été recueillies aux fins de surveillance en santé publique, elles n'ont pas à être approuvées par un comité d'examen éthique.

\section{Analyse statistique}

Les taux annuels et propres à l'âge ont été calculés à partir des estimations dans les populations provinciales et régionales tirées de la base de données BC Stats (http://www.bcstats.gov.bc.ca/Home.aspx). Les analyses ont été effectuées à l'aide de la version 9.3 du système SAS (SAS Inc., Cary, NC).

\section{Résultats}

\section{Tendances sur le plan de l'incidence globale à l'échelle provinciale}

Après des sommets épidémiques en 1996, 2000 et 2003, l'incidence de la coqueluche en C.-B. est passée à une activité endémique de faible niveau, pendant laquelle seule une activité périodique mineure a été signalée dans des régions géographiques limitées (p. ex. par la Interior Health Authority [IHA] en 2006 et en 2010, et par la Northern Health Authority et la Vancouver Island Health Authority [VIHA] en 2008) [figure 1]. L'incidence provinciale globale a atteint des niveaux historiquement bas de 3 cas pour 100000 habitants en 2010 et de 1 cas pour 100000 habitants en 2011, qui ont été suivis par une résurgence cyclique en 2012, au cours de laquelle l'incidence provinciale globale a atteint 10 cas pour 100000 habitants. Toutefois, le sommet d'incidence observé en 2012 est demeuré considérablement plus faible (moins de la moitié) que les sommets historiques observés en 1996 (23 cas pour 100000 habitants), en 2000 (38 cas pour 100000 habitants) et en 2003 (22 cas pour 100000 habitants) [figure 1]. 
Figure 1 : Incidence annuelle des cas confirmés de coqueluche en Colombie-Britannique pour l'ensemble de la province et les autorités sanitaires sélectionnées, de 1993 à 2013*

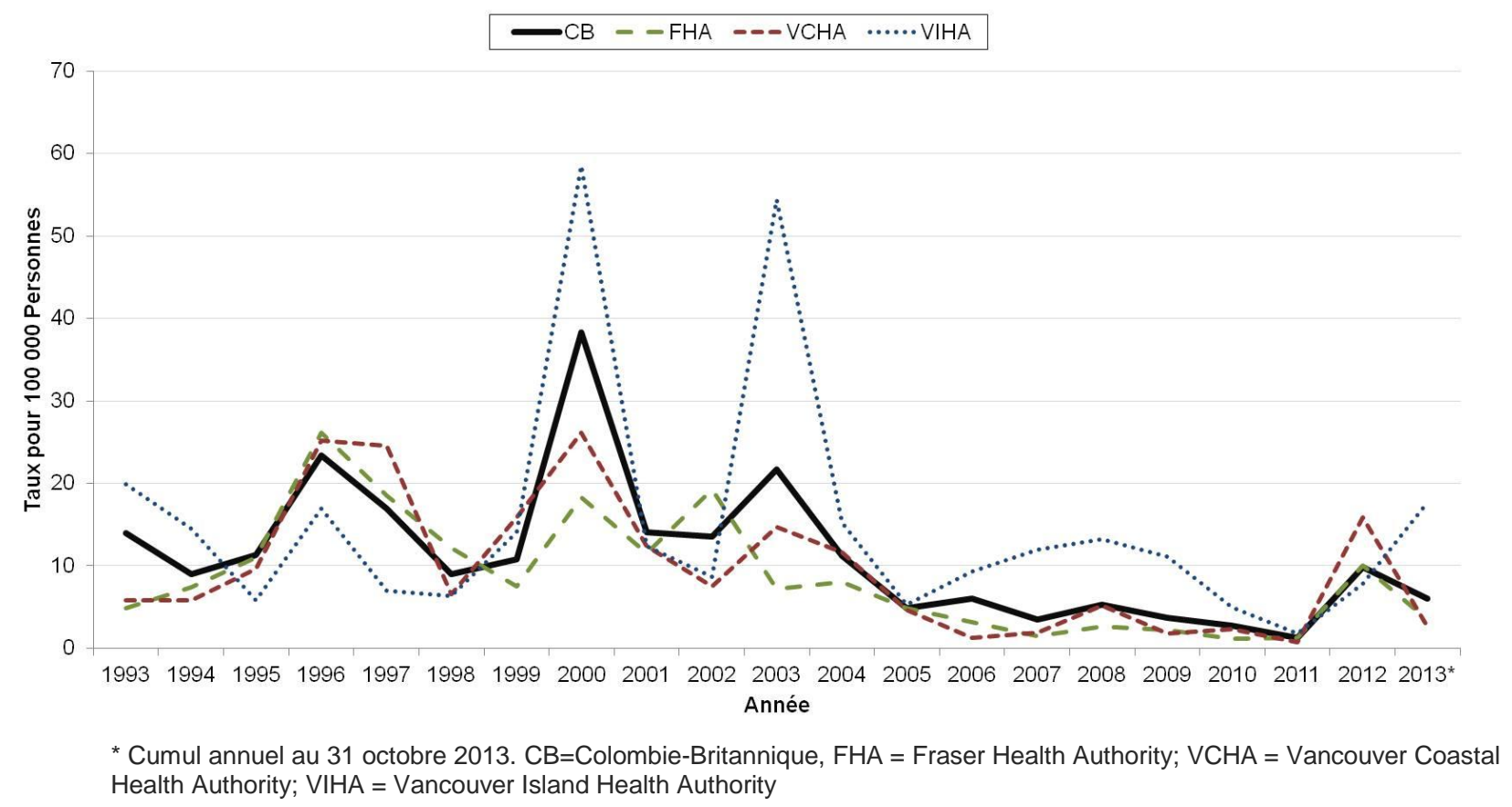

Par rapport à 2011, le PHMRL a reçu en 2012 un nombre de demandes de tests de dépistage de la coqueluche près de neuf fois plus élevé, soit une moyenne de plus de plus de 700 tests par mois en 2012 par rapport à environ 80 par mois en 2011; en moyenne, environ 300 tests ont été demandés chaque mois en 2013.

Cependant, les taux de tests positifs pour la coqueluche étaient comparables au cours de ces années et sont demeurés inférieurs à $5 \%$ au cours des périodes d'augmentation du volume des tests de dépistage entre février et avril 2012, en phase avec les autres causes prédominantes de maladies respiratoires au cours des mois d'hiver. Au cours des trois années, les taux de tests positifs pour la coqueluche ont atteint des sommets en juillet et en août, simultanément à des volumes de test plus faibles (figure 2). 
Figure 2 : Demandes de tests de dépistage de la coqueluche et pourcentage de résultats positifs pour la coqueluche du Laboratoire de santé publique et de référence en microbiologie de la Colombie-Britannique, selon le mois et l'année, de janvier 2011 à septembre 2013

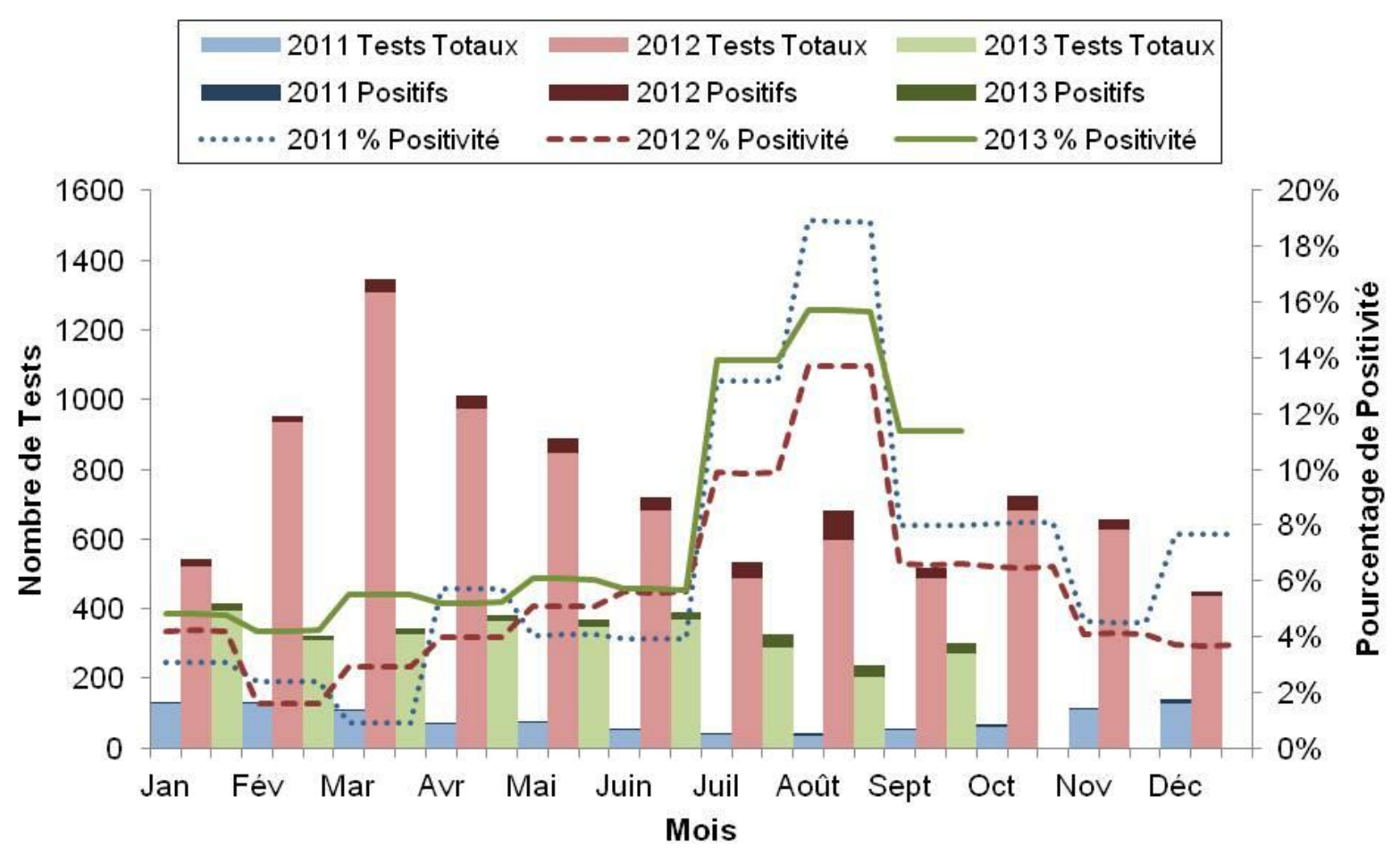

\section{Tendances sur le plan de l'incidence propre à l'âge à l'échelle provinciale}

En 2012, les taux d'incidence propre à l'âge étaient plus élevés chez les nourrissons de moins de 1 an (64 cas pour 100000 habitants) et les enfants de 12 et 13 ans (entre 56 et 57 cas pour 100000 habitants) [figure 3]. Les taux d'incidence les plus faibles ont été observés dans les groupes d'âge suivant immédiatement l'âge d'administration des doses de rappel du calendrier immunisation systématique, soit 16 cas pour 100000 enfants d'âge préscolaire (entre 1 et 4 ans) et 12 cas pour 100000 adolescents plus âgés (entre 16 et 19 ans) [figure 4A]. Des taux d'incidence légèrement plus élevés ont été observés chez les enfants d'âge scolaire plus jeunes (entre 5 et 9 ans, 28 cas pour 100000 habitants [figure 4A]), chez qui l'incidence propre à l'âge a augmenté graduellement chaque année supplémentaire à partir de l'âge d'administration de la dose de rappel prévue à la maternelle (figure 3). De même, l'incidence chez les adolescents âgés de 14 et 15 ans vers la période de l'administration de la dose de rappel prévue en 9e année était de 28 cas pour 100000 habitants (figure 4). Dans l'ensemble, l'incidence la plus faible en 2012 a été observée chez les adultes de 20 ans et plus (5 cas pour 100 000 habitants). 
Figure 3 : Incidence propre à l'âge des cas confirmés de coqueluche en Colombie-Britannique pour les années de sommet cyclique, 1993, 1996, 2000, 2003, 2012 et 2013 (cumul annuel)* $^{*}$

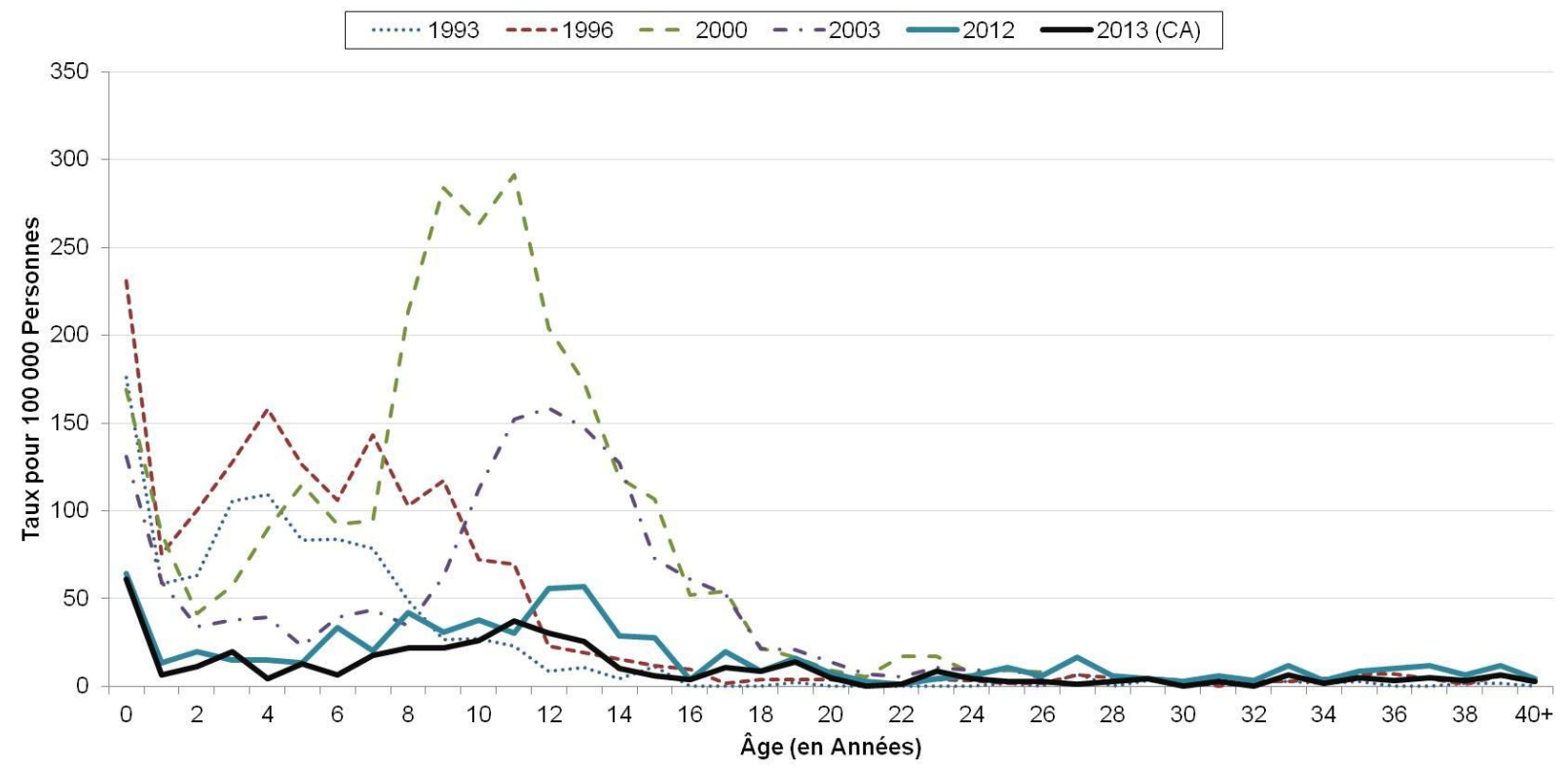

* Cumul annuel au 31 octobre 2013 
Figure 4 : A) Taux d'incidence et B) proportion de cas confirmés de coqueluche en Colombie-Britannique, par groupe d'âge, de 1993 à 2013*

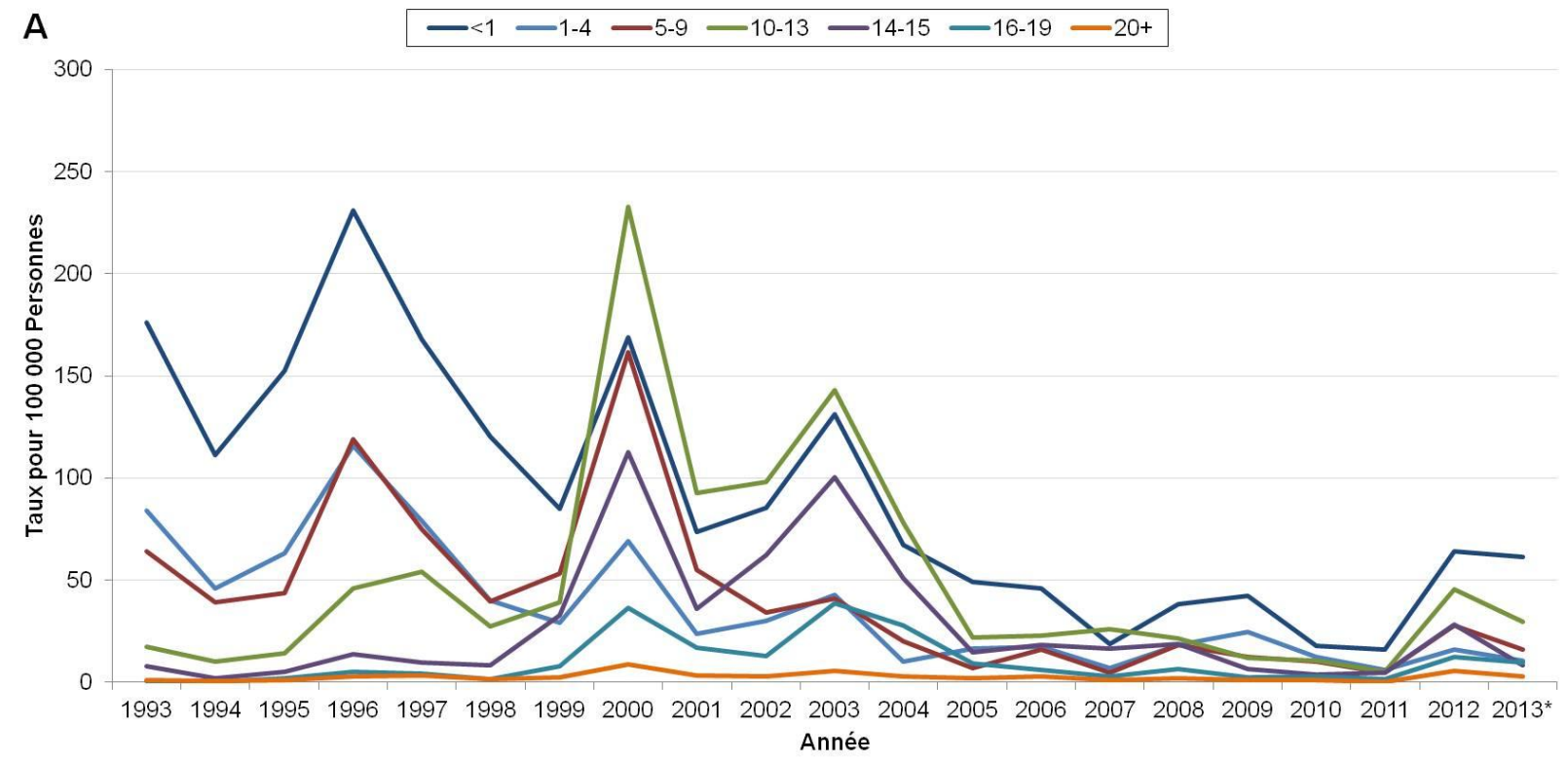

B

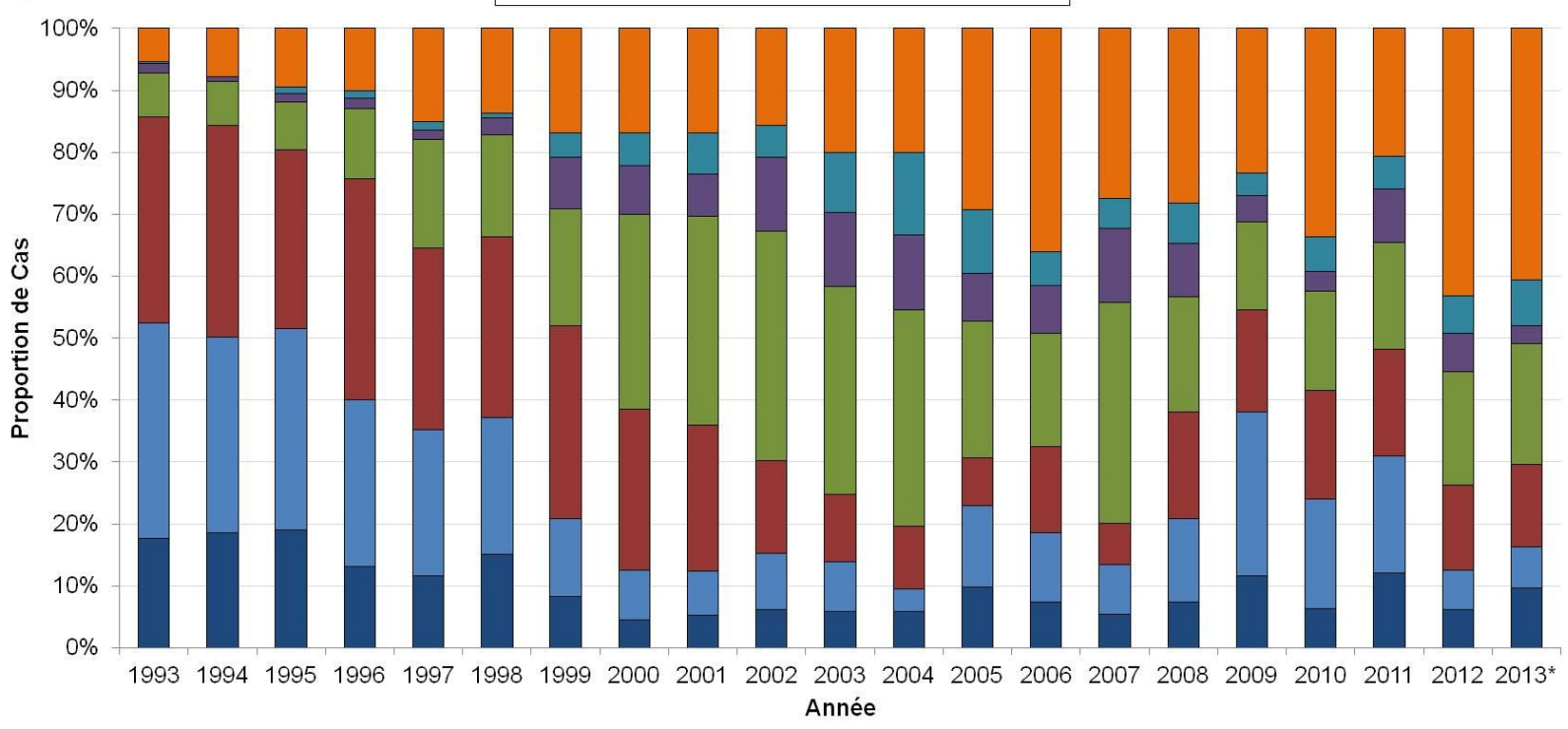

${ }^{*}$ Cumul annuel au 31 octobre 2013

Cela dit, les taux d'incidence plus élevés observés en C.-B. chez les préadolescents de 10 à 13 ans par rapport aux nourrissons de moins de 1 an, qui ont été notés initialement au cours de l'éclosion de l'année $2000^{7}$, se sont maintenus jusqu'en 2005, après quoi des taux plus élevés ont été observés chez les nourrissons. Les taux d'incidence de 64 cas pour 100000 nourrissons et de 46 cas pour 100000 préadolescents observés au cours du sommet de 2012 (figure 4A) illustrent cette différence. L'incidence plus faible chez les adolescents âgés de 16 à 19 ans (12 cas pour 100000 habitants) et de 14 et 15 ans (28 cas pour 100000 habitants) par rapport aux préadolescents âgés de 10 à 13 ans (46 cas pour 100000 habitants) observée en 2012 était déjà évidente au cours des années précédentes (marquées ou non par des épidémies), soit en 1996 (5, 14 et 47 cas pour 100000 habitants, respectivement), en 2000 (36, 113 et 233 cas pour 100000 habitants, respectivement) et en 2003 (39, 
100 et 143 par 100000 habitants, respectivement), une tendance considérablement antérieure à l'introduction de la dose de rappel pour les adolescents de la 9e année, en 2004.

Dans tous les groupes d'âge chez les enfants, l'incidence au cours du sommet cyclique de 2012 a été de trois à six fois inférieure par rapport aux sommets d'activité observés au cours des 20 années précédentes.

Inversement, l'incidence chez les adultes de 20 ans et plus est demeurée à des niveaux faibles plus stables, soit 5 cas signalés par 100000 habitants en 2012, ce qui représente une diminution de moins de deux fois par rapport au sommet d'activité historique de 9 cas pour 100000 habitants observé en 2000. Toutefois, en raison de la baisse considérable de l'incidence dans tous les groupes d'âge chez les enfants, les adultes âgés de 20 ans et plus représentent une proportion en augmentation constante de l'ensemble des cas (figure 4B).

\section{Éclosions régionales, 2012 et 2013}

L'éclosion survenue en 2012 en C.-B. était largement concentrée dans la région des basses-terres continentales, où l'incidence globale a été de 10 cas pour 100000 habitants signalés à la FHA et de 16 cas pour 100000 habitants signalés à la VCHA, ce qui représente des taux inférieurs aux sommets cycliques observés précédemment dans ces régions (figure 1). Des zones localisées d'activité plus intense ont largement contribué à la variation des taux à l'échelle régionale. Dans la région de Fraser East relevant de la FHA, l'incidence de la coqueluche a atteint 24 cas pour 100000 habitants, alors que dans la VCHA, l'incidence dans les régions de North Shore/Coast Garibaldi a atteint 36 cas pour 100000 habitants (figure 5). Une activité plus intense a été associée à des éclosions localisées dans des écoles ou d'autres regroupements sociaux, ainsi qu'à des groupes de faible taux de vaccination.

Figure 5 : Incidence des cas confirmés de coqueluche en Colombie-Britannique, selon la zone de prestation de services de santé, en 2012 et $2013^{*}$

2012

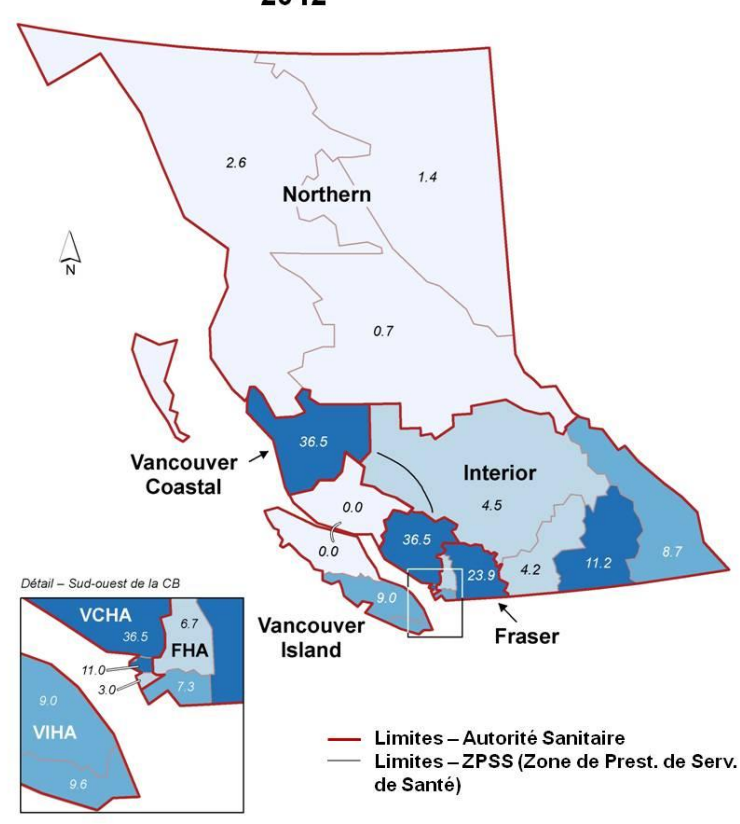

2013

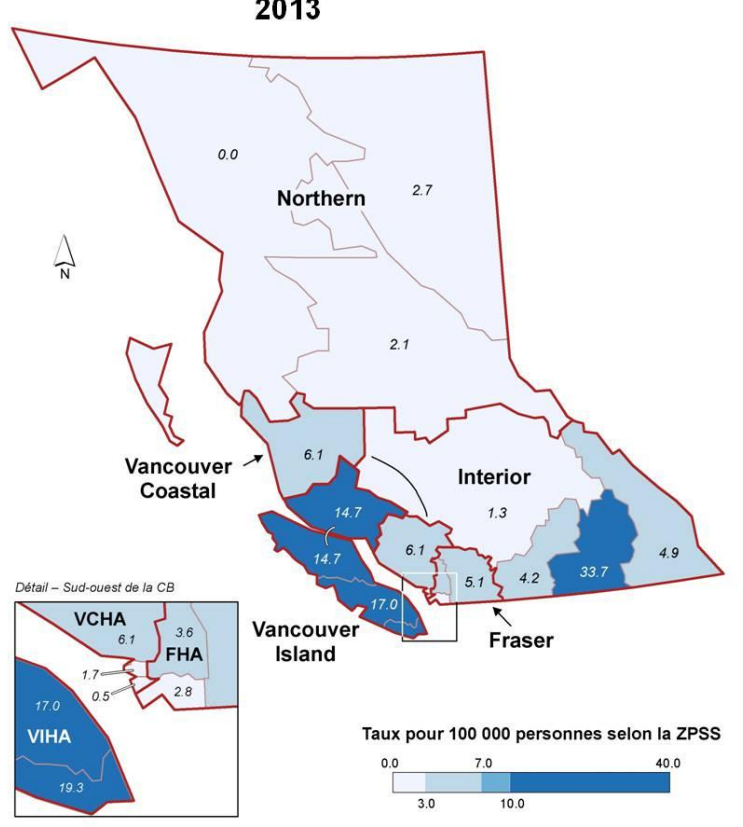

${ }^{*}$ Cumul annuel au 31 octobre 2013 
Depuis janvier 2013, les taux d'incidence dans la FHA et la VCHA sont revenus aux niveaux endémiques prévus. Les taux d'incidence cumulatifs globaux pour 2013 étaient de $\leq 5$ cas pour 100000 habitants dans chacune de ces autorités régionales de la santé, ce qui est comparable aux taux observés au cours des années précédentes qui n'ont pas connu d'épidémies et dans l'ensemble de la C.-B. (figure 1). À l'opposé, un sommet asynchrone de l'activité de la coqueluche a été observé dans la VIHA en 2013. Le taux d'incidence cumulatif dans cette région, qui était de 18 cas pour 100000 habitants, est légèrement supérieur aux taux observés pour l'ensemble de l'année au cours des récentes périodes de sommets d'activité observées de 2007 à 2009 (qui variaient entre 11 et 13 cas pour 100000 habitants), mais est environ trois fois inférieur aux sommets historiques observés en 2000 (59 cas pour 100000 habitants) et en 2003 (54 cas pour 100000 habitants) [figure 1]. L'activité observée dans la VIHA en 2013 est également attribuable à des zones localisées d'intensité supérieure. Des éclosions localisées dans les collectivités limitrophes de Kootenay, dans la IHA, où l'incidence a atteint 34 cas pour 100000 habitants, ont également été signalées en 2013 (figure 5).

Au cours des éclosions survenues en 2012 dans la VCHA et la FHA, moins de la moitié des cas observés chez les enfants âgés de 2 mois à 20 ans avaient reçu tous les vaccins prévus en fonction de l'âge dans l'ensemble; une proportion importante était soit partiellement immunisée ou non immunisée (environ $25 \%$ dans chaque catégorie), ou l'état vaccinal était inconnu (4\%). Il y a eu six cas d'hospitalisation parmi les cas signalés dans ces régions, dont deux admissions à l'unité de soins intensifs. Tous les cas d'hospitalisation, sauf un, concernaient des nourrissons âgés de moins de 3 mois. Trois autres hospitalisations ont été signalées ailleurs dans la province au cours de l'année 2012, soit deux nourrissons de moins de 12 mois et un nourrisson de moins de 3 mois qui a été admis à l'unité de soins intensifs. En 2012, le taux confirmé d'hospitalisations en raison de la coqueluche chez des nourrissons dans les régions touchées par les éclosions et dans l'ensemble de la province a été de 18 cas pour 100000 habitants. Bien qu'aucun décès n'ait été signalé en 2012, un décès est survenu en 2013 chez un nourrisson immunisé âgé de moins de 3 mois. II s'agissait du premier décès attribuable à la coqueluche identifié en C.-B. depuis 2005.

\section{Discussion}

Ce résumé de la surveillance décrit les tendances confirmées des cas de coqueluche en C.-B. sur une période de 20 ans, soit de 1993 à 2013. Au cours de cette période, l'activité de la coqueluche a présenté les fluctuations cycliques attendues tous les 2 à 5 ans. Les signalements de cas confirmés de coqueluche ont augmenté au cours des années 1990 pour atteindre un sommet en 2000, suivi par une diminution des signalements au cours de la décennie suivante. Après des niveaux historiquement bas en 2010 et en 2011, un autre sommet cyclique, mais moins important, a été observé en 2012. Le changement de la répartition selon l'âge, soit une augmentation du nombre de préadolescents et une diminution du nombre d'enfants d'âge préscolaire, qui a été souligné initialement au cours de l'éclosion de l'année 2000 en C.-B. ${ }^{7}$, s'est poursuivi tout au long de la plus récente période d'activité maximale.

Durant l'ère prévaccinale, le sommet d'incidence était observé chez les enfants d'âge préscolaire et moins de 20 $\%$ des cas survenaient chez des nourrissons ${ }^{7}$. Depuis l'avènement de la vaccination, on observe maintenant un sommet d'incidence chez les nourrissons de moins de 1 an, suivi d'un deuxième sommet chez les enfants d'âge préscolaire, ainsi qu'une diminution de l'incidence globale. Depuis 2000, un autre changement lié à l'âge est également observé : bien que les nourrissons de moins de 1 an présentent toujours un risque élevé, le deuxième sommet survient maintenant chez les préadolescents et les adolescents au lieu des enfants d'âge préscolaire. Au cours des derniers sommets survenus en 2012 et en 2013, cette tendance sur le plan de l'âge était toujours observable. Toutefois, l'augmentation prononcée de l'incidence observée chez les préadolescents au cours des éclosions de 2000 et de 2003 a fait place à une transition relativement plus graduelle des enfants d'âge scolaire plus jeunes à ceux plus âgés, simultanément à une activité globale plus faible.

L'introduction du test diagnostique basé sur la réaction en chaîne de la polymérase (RCP) pourrait avoir contribué à l'augmentation spectaculaire du nombre de signalements de cas de coqueluche observée en C.-B. vers la fin des années 1990 et au début des années 2000. La RCP est considérée comme un test diagnostique plus sensible que le test diagnostique basé sur la culture puisqu'elle offre une période de détection accrue, soit six 
semaines suivant l'apparition de la toux, bien que la sensibilité soit optimale au cours des deux premières semaines $^{18-20}$. Plus particulièrement, la RCP pourrait avoir permis d'accroître le dépistage chez les enfants plus âgés et les adultes qui pourraient ne pas avoir été diagnostiqués au moyen des techniques de culture standard en raison de la période écoulée avant la consultation en raison d'une toux prolongée et non spécifique. La baisse de nombre de signalements des cas de coqueluche dans l'ensemble et dans tous les groupes d'âge depuis l'année 2000, malgré la disponibilité du test diagnostique de la RCP, devra être expliquée autrement.

Les épidémies de 2000 et de 2003, qui ont été accompagnées par un changement dans la répartition de l'âge, soit une augmentation chez les enfants plus âgés, ont été expliquées précédemment par un effet de cohorte combinant une susceptibilité initiale accumulée et une diminution subséquente de l'immunité chez les receveurs du vaccin absorbé à germes entiers contre la coqueluche, qui était moins efficace ${ }^{5 ; 7 ; 12}$. Au cours des 15 années suivant l'intégration du vaccin acellulaire au programme d'immunisation systématique chez les enfants, une proportion de plus en plus élevée d'enfants font partie des cohortes de naissances qui n'ont reçu que le vaccin acellulaire à chacune de leurs cinq doses administrées en primovaccination. En 2012 et en 2013, les adolescents de 14 et 15 ans et les enfants plus jeunes auraient formé les premières cohortes d'enfants entièrement immunisés par le vaccin acellulaire seulement. Bien que la coqueluche demeure bien maîtrisée en C.-B. en comparaison avec les niveaux d'activité historiques, il est trop tôt pour évaluer les répercussions à long terme potentielles des changements apportés au programme d'immunisation systématique sur le fardeau de la coqueluche.

En plus des changements apportés au programme d'immunisation, les faibles niveaux d'activité de la coqueluche en C.-B. pourraient également refléter les changements survenus dans l'immuno-épidémiologie de la population à la suite d'épidémies importantes survenues dans la province en 1996, et 2000 et en 2003. À l'ère prévaccinale, presque tous les enfants auraient été exposés à la coqueluche avant l'âge de 12 ans ${ }^{7}$. II reste à déterminer si le vaccin acellulaire peut maintenir une protection en l'absence de la stimulation immunitaire étendue procurée par l'infection naturelle, qui semble assurer une protection plus durable que l'immunité acquise par vaccin. De récentes études d'observation menées pendant des éclosions survenues aux États-Unis ont mis en évidence la diminution rapide de la protection du vaccin acellulaire au cours des 2 à 5 ans suivant la 5 e dose de rappel ${ }^{21-24}$. Le profil de risque lié à l'âge mis en évidence dans nos données de surveillance ainsi que l'augmentation graduelle de l'incidence entre les âges de 5 et 13 ans corroborent la protection incomplète attribuable à la diminution de l'immunité. Toutefois, ces données ne permettent pas de déterminer si l'introduction de la dose de rappel chez les adolescents au programme d'immunisation provincial en 2004 a permis d'atténuer l'avancement de l'effet de cohorte observé chez les préadolescents et les adolescents. D'autres ont suggéré que les changements observés dans l'incidence par tranche d'âge seraient plutôt attribuables aux différences dans les réseaux sociaux et les profils de fréquentation sociale en fonction du groupe d'âge ${ }^{25}$. La tendance persistante consistant en une réduction de l'incidence chez les adolescents plus âgés, qui est apparue avant les changements récents apportés aux programmes d'immunisation, pourrait appuyer cette affirmation, toutefois d'autres facteurs, comme les différences liées à l'âge sur le plan de la gravité de la maladie ou les comportements en matière de sollicitation des soins, pourraient également expliquer ce constat. Compte tenu des niveaux d'activité actuels de la coqueluche en C.-B. et des preuves de plus en plus nombreuses de la diminution de l'immunité après l'administration des doses de rappel d'un vaccin acellulaire, la valeur des doses supplémentaires et le moment d'administration optimale demandent une évaluation plus poussée.

\section{Limites}

Les limites de la surveillance de la coqueluche en C. B., comme partout ailleurs, comprennent les biais liés au signalement et au mauvais classement. Seuls les cas confirmés décelés dans le cadre de la surveillance passive de routine ont été pris en compte dans le présent résumé, ce qui signifie que l'incidence réelle est sous-estimée. Puisque des tests diagnostiques basés sur la réaction en chaîne de la polymérase sont systématiquement mis en œuvre en C.-B. depuis 1998, les profils d'incidence observés au fil du temps depuis cette mise en œuvre, principalement une baisse substantielle des taux, reflètent probablement des tendances précises, voire un risque absolu. II est probable que la diminution de l'incidence au fil du temps au sein des différents groupes d'âge soit également robuste, mais les comparaisons entre les groupes d'âge pourraient être moins fiables. Puisque les jeunes nourrissons présentent des symptômes plus traditionnels et plus graves de la maladie, les cliniciens 
pourraient être plus susceptibles d'effectuer des tests, de poser un diagnostic ou de signaler les cas de coqueluche dans ce groupe d'âge. Les symptômes atypiques de la maladie chez les enfants immunisés, les adolescents plus âgés et les adultes, pourraient avoir contribué, en partie, à la faible incidence observée. Les hospitalisations ont été fondées sur les cas de coqueluche confirmés exclusivement dans le cadre de la surveillance accrue mise en place au cours de l'éclosion de 2012. Le taux d'hospitalisation de 18 cas pour 100 000 habitants est inférieur aux estimations fondées sur le diagnostic clinique extraites des registres administratifs de congés des hôpitaux au cours des sommets observés précédemment en C.-B. (qui variaient entre 50 et 70 cas pour 100000 habitants) $)^{3}$. Ces données n'étaient pas facilement accessibles aux fins de l'analyse actuelle. Bien que le premier ensemble de données puisse produire une sous-estimation, il est plus probable que le deuxième ensemble de données sous-estime les taux d'hospitalisation attribuables à la coqueluche. Finalement, l'asynchronie du moment et du lieu géographique des sommets cycliques n'est pas inattendue Le regroupement des données de surveillance à l'échelle de l'autorité régionale ou provinciale pourrait masquer une activité plus intense ou plus discrète au niveau local.

\section{Conclusions}

Après des épidémies importantes en 1996, 2000 et 2003, la C.-B. a connu des taux historiquement bas d'activité de la coqueluche en 2010 et 2011, qui ont été suivis en 2012 par des éclosions à l'échelle régionale concentrées dans les régions des basses-terres continentales de la province. En 2013, l'activité de la coqueluche était revenue à des niveaux endémiques dans ces régions, mais une activité asynchrone était localisée sur l'île de Vancouver et dans certaines régions de l'intérieur. Au cours des sommets cycliques observés depuis 2000, l'incidence la plus élevée a été signalée chez les nourrissons et les préadolescents, alors que l'incidence la plus faible a été signalée chez les adultes. Les épidémies récentes et les changements apportés au programme d'immunisation provincial pourraient avoir modifié les tendances sur le plan de l'immunité liée à l'âge, tandis que des différences sur le plan des réseaux de contacts sociaux pourraient modifier le risque d'exposition, ce qui se reflète dans les tendances actuelles observées. Malgré les limites connues sur le plan de la surveillance, un suivi continu ainsi que des études actives sur le risque lié à l'âge et l'efficacité des vaccins sont nécessaires et devraient être encouragés afin de déterminer quelles modifications pourraient éventuellement être apportées aux politiques et aux programmes provinciaux.

\section{Références}

1. Broutin H, Guegan JF, Elguero E, Simondon F, Cazelles B. Large-scale comparative analysis of pertussis population dynamics: periodicity, synchrony, and impact of vaccination. Am J Epidemiol. 2005;161(12):115967.

2. Bettinger JA, Halperin SA, De Serres G, Scheifele DW, Tam T. The effect of changing from whole-cell to acellular pertussis vaccine on the epidemiology of hospitalized children with pertussis in Canada. Pediatr Infect Dis J. 2007;26(1):31-5.

3. Skowronski DM, Janjua NZ, Tsafack EP, Ouakki M, Hoang L, De Serres G. The number needed to vaccinate to prevent infant pertussis hospitalization and death through parent cocoon immunization. Clin Infect Dis. 2012;54(3):318-27.

4. Agence de la santé publique du Canada. Partie 4. Vaccins actifs. : Guide canadien d'immunisation. Ottawa : Agence de la santé publique du Canada; 2012

5. Galanis E, King AS, Varughese P, Halperin SA. Changing epidemiology and emerging risk groups for pertussis. CMAJ. 2006;174(4):451-2.

6. Tam TW, Bentsi-Enchill A. The return of the 100-day cough: resurgence of pertussis in the 1990s. CMAJ. 1998;159(6):695-6.

7. Skowronski DM, De Serres G, MacDonald D, Wu W, Shaw C, Macnabb J, et al. The changing age and seasonal profile of pertussis in Canada. J Infect Dis. 2002;185(10):1448-53.

8. Bentsi-Enchill AD, Halperin SA, Scott J, Maclsaac K, Duclos P. Estimates of the effectiveness of a whole-cell pertussis vaccine from an outbreak in an immunized population. Vaccine. 1997;15(3):301-6. 
9. De Serres G, Boulianne N, Duval B, Dery P, Rodriguez AM, Masse R, et al. Effectiveness of a whole cell pertussis vaccine in child-care centers and schools. Pediatr Infect Dis J. 1996;15(6):519-24.

10. Gustafsson L, Hallander HO, Olin P, Reizenstein E, Storsaeter J. A controlled trial of a two-component acellular, a five-component acellular, and a whole-cell pertussis vaccine. N Engl J Med. 1996;334(6):349-55.

11. Halperin SA, Bortolussi R, MacLean D, Chisholm N. Persistence of pertussis in an immunized population: results of the Nova Scotia Enhanced Pertussis Surveillance Program. J Pediatr. 1989;115(5 Pt 1):686-93.

12. Ntezayabo B, De Serres G, Duval B. Pertussis resurgence in Canada largely caused by a cohort effect. Pediatr Infect Dis J. 2003;22(1):22-7.

13. Zhang L, Prietsch SO, Axelsson I, Halperin SA. Acellular vaccines for preventing whooping cough in children. Cochrane Database Syst Rev. 2012;3:CD001478.

14. Comité consultatif national de l'immunisation (CCNI). Prévention de la coqueluche chez les adolescents et les adultes. Relevé des maladies transmissibles au Canada. 2003;29(DCC-5):1-9.

15. Cherry JD. Epidemic pertussis in 2012 - the resurgence of a vaccine-preventable disease. N Engl J Med. 2012;367(9):785-7.

16. California Department of Public Health. Pertussis report. May 16, 2011 [en ligne]. Sacramento: California Department of Public Health; 2011 [consulté le 18 octobre 2013]. http://www.cdph.ca.gov/programs/immunize/Pages/PertussisSummaryReports.aspx

17. BC Centre for Disease Control. Management of specific diseases - pertussis. In: British Columbia Communicable Disease Manual. Vancouver: BC Centre for Disease Control, 2010. p. 1-36.

18. He Q, Schmidt-Schlapfer G, Just M, Matter HC, Nikkari S, Viljanen MK, et al. Impact of polymerase chain reaction on clinical pertussis research: Finnish and Swiss experiences. J Infect Dis. 1996;174(6):1288-95.

19. Sotir MJ, Cappozzo DL, Warshauer DM, Schmidt CE, Monson TA, Berg JL, et al. Evaluation of polymerase chain reaction and culture for diagnosis of pertussis in the control of a county-wide outbreak focused among adolescents and adults. Clin Infect Dis. 2007;44(9):1216-9.

20. van der Zee A, Agterberg C, Peeters M, Mooi F, Schellekens J. A clinical validation of Bordetella pertussis and Bordetella parapertussis polymerase chain reaction: comparison with culture and serology using samples from patients with suspected whooping cough from a highly immunized population. J Infect Dis. 1996;174(1):89-96.

21. Klein NP, Bartlett J, Rowhani-Rahbar A, Fireman B, Baxter R. Waning protection after fifth dose of acellular pertussis vaccine in children. N Engl J Med. 2012;367(11):1012-9.

22. Misegades LK, Winter K, Harriman K, Talarico J, Messonnier NE, Clark TA, et al. Association of childhood pertussis with receipt of 5 doses of pertussis vaccine by time since last vaccine dose, California, 2010. JAMA. 2012;308(20):2126-32.

23. Tartof SY, Lewis M, Kenyon C, White K, Osborn A, Liko J et al. Waning immunity to pertussis following 5 doses of DTaP. Pediatrics. 2013;131(4):e1047-52.

24. Witt MA, Katz PH, Witt DJ. Unexpectedly limited durability of immunity following acellular pertussis vaccination in preadolescents in a North American outbreak. Clin Infect Dis. 2012;54(12):1730-5.

25. Rohani $P$, Zhong $X$, King AA. Contact network structure explains the changing epidemiology of pertussis. Science. 2010;330(6006):982-5.

\section{Remerciements}

Les auteurs souhaitent remercier les personnes et les organisations rattachées aux autorités sanitaires pour leurs contributions aux données de surveillance. Plus particulièrement, nous aimerions remercier les personnes suivantes : le Dr Mark Bigham et Stephanie Konrad (Fraser Health Authority), Sara Forsting (Vancouver Coastal Health Authority), le Dr Dee Hoyano (Vancouver Island Health Authority) et Yin Chang (Laboratoire de santé publique et de référence en microbiologie, Provincial Health Services Authority). 


\section{Conflit d'intérêts}

II n'y a aucun conflit d'intérêts à déclarer.

\section{Financement}

Aucun 